\title{
Towards On-Chip Net-Gain in CMOS-compatible Waveguides
}

\author{
Clemens J. Krückel, Peter A. Andrekson, and Victor Torres-Company \\ Department of Microtechnology and Nanoscience (MC2), Chalmers University of Technology, SE-412 96 Gothenburg, Sweden
}

On-chip optical parametric gain was first demonstrated in a silicon waveguide using a pulsed pump source [1]. However, the realization of optical net-gain with a continuous-wave $(\mathrm{CW})$ pump remains a challenge in integrated platforms. Highly nonlinear fibers can provide parametric gain with CW pump [2]. The key advantage of this lowloss platform is its long effective length $\mathrm{L}_{\text {eff, }}$ which results in a huge nonlinear phase shift, $\Theta_{\mathrm{NL}}=\gamma \mathrm{PL}_{\mathrm{eff}} \mathrm{using}$ moderate power levels, $\mathrm{P}$, in spite of the relatively low nonlinear parameter $\gamma$. The question we address in this contribution is: How far are we from obtaining net gain in a CMOS-compatible platform under CW pumping?

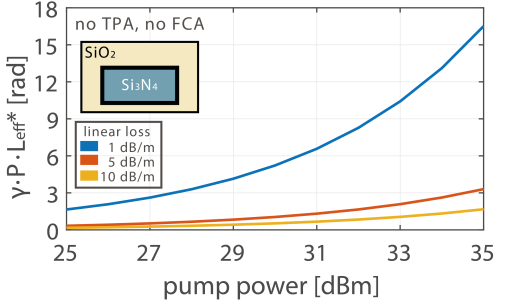

(a)

Table: Required parameters for $\sim 10 \mathrm{~dB}$ optical net-gain

\begin{tabular}{|c|c|c|c|}
\hline & $\mathrm{Si}_{3} \mathrm{~N}_{4}$ & $\mathrm{Si}$ & $\mathrm{Si}+\operatorname{pin}$ \\
\hline Linear loss & 5 & 1.3 & 33 \\
\hline Dispersion [ $\mathrm{ps} / \mathrm{nm} / \mathrm{km}]$ & 8 & 12 & 180 \\
\hline Pump power [dBm] & 35 & 13 & 35 \\
\hline Physical length [m] & 1.4 & 1.9 & 0.055 \\
\hline$\left[(\mathrm{Wm})^{-1}\right]$ & 1.2 & 300 & 200 \\
\hline [ps] & n. a. & 800 & 10 \\
\hline
\end{tabular}
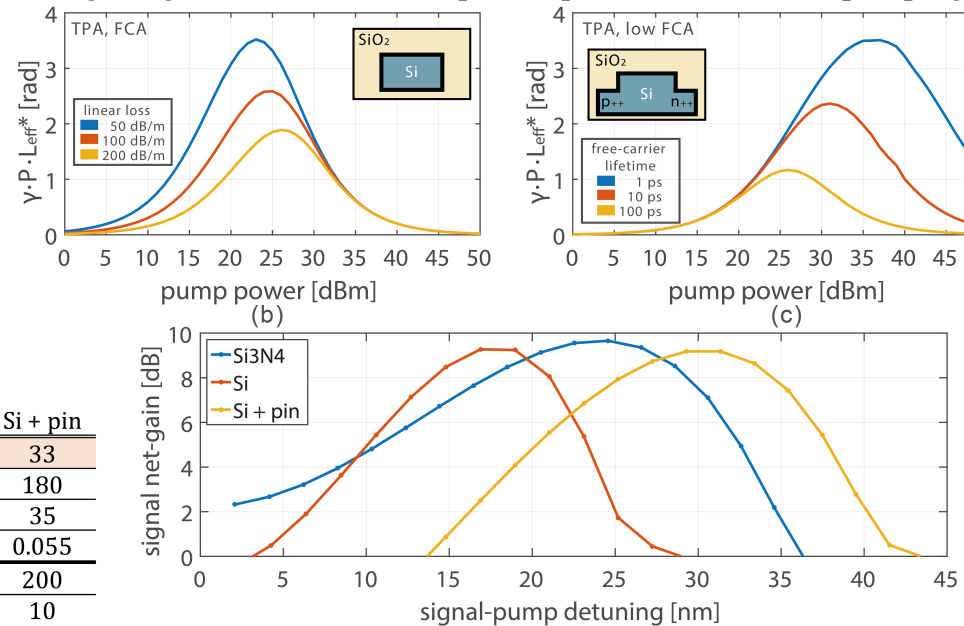

(d)

Fig. 1 Nonlinear phase shift vs. pump power in (a) $\mathrm{Si}_{3} \mathrm{~N}_{4}$ (no TPA, no FCA). (b) Si (TPA, FCA with $\tau 800$ ps) and (c) Si with carrier removal (TPA, low FCA, linear loss of $100 \mathrm{~dB} / \mathrm{m}$ [3]). For all three cases, we consider $\mathrm{L}_{\text {eff }}{ }^{*}$ as the propagation length at which the pump power decays by $4.3 \mathrm{~dB}$. Note that this magnitude becomes power-dependent for the structures shown in (b) and (c). (d) Signal net-gain vs. signal-pump detuning. The GVD was chosen to cover a similar gain spectrum in the three cases. The signal power is chosen to be $-60 \mathrm{dBm}$.

We focus on high-confinement strip waveguides based on silicon nitride $\left(\mathrm{Si}_{3} \mathrm{~N}_{4}\right)$ [4] (that can achieve low propagation losses and have moderate nonlinear coefficient) and silicon ( $\mathrm{Si}$ ) nanowires [5] (that can achieve high nonlinear coefficient but have relatively high linear propagation losses). We restrict the study to the $1.5 \mu \mathrm{m}$ region. As silicon displays nonlinear losses (TPA and FCA), we also present a ridge waveguide design that reduces FCA by free carrier removal [6]. In the upper row figures, we analyze the nonlinear phase shift as a function of power. The nonlinear parameter is kept at a reasonable value with today's technology (see Table). In absence of nonlinear loss, the nonlinear phase shift scales linearly with optical pump power, with a slope increasing for lower linear losses. The silicon waveguide shows nonlinear loss at higher power levels (TPA coefficient of $0.7 \mathrm{~cm} / \mathrm{GW}, \mathrm{FCA}$ coefficient of $\left.1.45 \cdot 10^{-17}(\lambda / 1.55)^{2} \cdot \Delta \mathrm{N}[6]\right)$. This results in an optimum launched power that maximizes the nonlinear phase shift. This maximum can be increased and shifted to lower power levels by reducing the propagation losses. In a waveguide with carrier removal, a reverse biased pin-diode reduces the free-carrier lifetime, which gives another tuning parameter to increase the nonlinear phase shift. In Fig. 1.c this is illustrated for three different $\tau$ and constant propagation losses of $1 \mathrm{~dB} / \mathrm{cm}$.

In Fig. 1d, we analyze the minimum amount of linear loss that would be necessary in order to achieve $10 \mathrm{~dB}$ net gain. For $\mathrm{Si}$, we choose the power that maximizes the nonlinear phase shift and for the pin diode structure we select 10 ps of carrier lifetime, similar to state of the art structures [6]. Our conclusion is clear: linear loss is the major challenge to overcome in order to obtain parametric net gain. With today's technology, we envision it is more realistic to achieve net gain in silicon nitride waveguides than in silicon structures. Silicon nitride microresonators with low anomalous dispersion and losses equivalent to $<1 \mathrm{~dB} / \mathrm{m}$ have been fabricated [7]. The challenge to fabricate spiral structures with a physical length of $1 \mathrm{~m}$ seems to us more feasible than decreasing the propagation losses of Si pin diode structures by an order of magnitude in a platform that simultaneously displays anomalous dispersion [3].

\section{References}

[1] Foster et al., "Broadband optical parametric gain on a silicon photonic chip," Nature, 441, 960-963 (2006)

[2] Hansryd et al., "Fiber-based optical parametric amplifiers and their ...," IEEE J. Sel. Topics Quantum Electron., 8, 506-520 (2002)

[3] Da Ros et al., "Phase regeneration of DPSK signals in a silicon waveguide with reverse-biased p-i-n ...," Op. Exp., 22, 5029-5036 (2014)

[4] Moss et al., "New CMOS-compatible platforms based on silicon nitride and Hydex for nonlinear,..." Nature Photon., 7, 597-607 (2013).

[5] Leuthold et al., "Nonlinear silicon photonics," Nature Photon., 4, 535-544 (2010)

[6] Turner-Foster et al., "Ultrashort free-carrier lifetime in low-loss silicon nanowaveguides," Op. Exp., 18, 3582-3591 (2010)

[7] Ji et al., "Breaking the Loss Limitations of On-chip High-confinement Resonators," arXiv:1609.08699 (2016) 